\title{
Contribution of proteomics to understanding the role of tumor-derived exosomes in cancer progression: State of the art and new perspectives
}

\author{
Fontana Simona, Saieva Laura, Taverna Simona and Alessandro Riccardo \\ Dipartimento di Biopatologia e Biotecnologie Mediche e Forensi, Sezione di Biologia e Genetica, Università di \\ Palermo, Palermo, Italy
}

\begin{abstract}
Exosomes are nanometer-sized vesicles (40-100 $\mathrm{nm}$ diameter) of endocytic origin released from different cell types under both normal and pathological conditions. They function as cell free messengers, playing a relevant role in the cell-cell communication that is strongly related to the nature of the molecules (proteins, mRNAs, miRNAs, and lipids) that they transport. Tumor cells actively shed exosomes into their surrounding microenvironment and growing evidence indicates that these vesicles have pleiotropic functions in the regulation of tumor progression, promoting immune escape, tumor invasion, neovascularization, and metastasis. During the last few years remarkable efforts have been made to obtain an accurate definition of the protein content of tumor-derived exosomes (TDEs) by applying MS-based proteomic technologies. To date, TDEs proteomic studies have been mainly utilized to catalog TDEs proteins with the purpose of identifying disease biomarkers. The future challenge for improving our understanding and characterization of TDEs will be the implementation of new systemsdriven and proteomic integrative strategies. The aim of this article is to provide an overview of the most characterized exosomes-mediated mechanisms that contribute to the pathogenesis of cancer and to review recent proteomics data that support the protumorigenic role of TDEs.
\end{abstract}

\section{Keywords:}

Cell biology / Tumor-derived exosome / Tumor progression

\section{Introduction}

Cell-to-cell communication in a living multicellular organism is an essential process that promotes and coordinates the activities among different groups of cells, creating a cooperative whole. Classically, cells are thought to communicate with each other via direct contact (juxtacrine signaling) or via soluble mediators (autocrine, paracrine, and endocrine signaling). However, during the last few years an understanding of

Correspondence: Dr. Simona Fontana, Dipartimento di Biopatologia e Biotecnologie Mediche e Forensi, Sezione di Biologia e Genetica, Università di Palermo, via Divisi, 83, 90133 Palermo, Italy E-mail: simona.fontana@unipa.it

Fax: +39-091-6554624

Abbreviations: CML, chronic myelogenous leukemia; ECM, extracellular matrix; ILV, intraluminal vesicle; MMP, matrix metalloproteinase; NK cells, natural killer cells; TDE, tumor-derived exosomes
Received: August 31, 2012

Revised: October 1, 2012

Accepted: October 2, 2012 the function of membrane-limited vesicles released by cells in transferring small packages of information to target cells is rapidly emerging. Among them, exosomes seem to play a relevant role in trans signaling to neighboring cells.

Exosomes are nanometer-sized vesicles that represent a distinct class of membrane vesicles (40-100 nm diameter) of endocytic origin that are released from different cell types under both normal and pathological conditions [1,2]. In addition to cultured cells, exosomes have further been isolated from a number of body fluids such as plasma [3], urine [4], synovial fluid [5], malignant effusions [6], epididymal fluid [7], and from seminal plasma [8].

Exosomes are derived from the intraluminal vesicles (ILVs) of multivesicular bodies (MVBs), formed through the reversed budding of the limiting membrane of late endosomes. During biogenesis, ILVs encapsulate material from cytoplasm including RNA (both mRNA and microRNA) and

Colour Online: See the article online to view Figs. 1-3 in colour. 


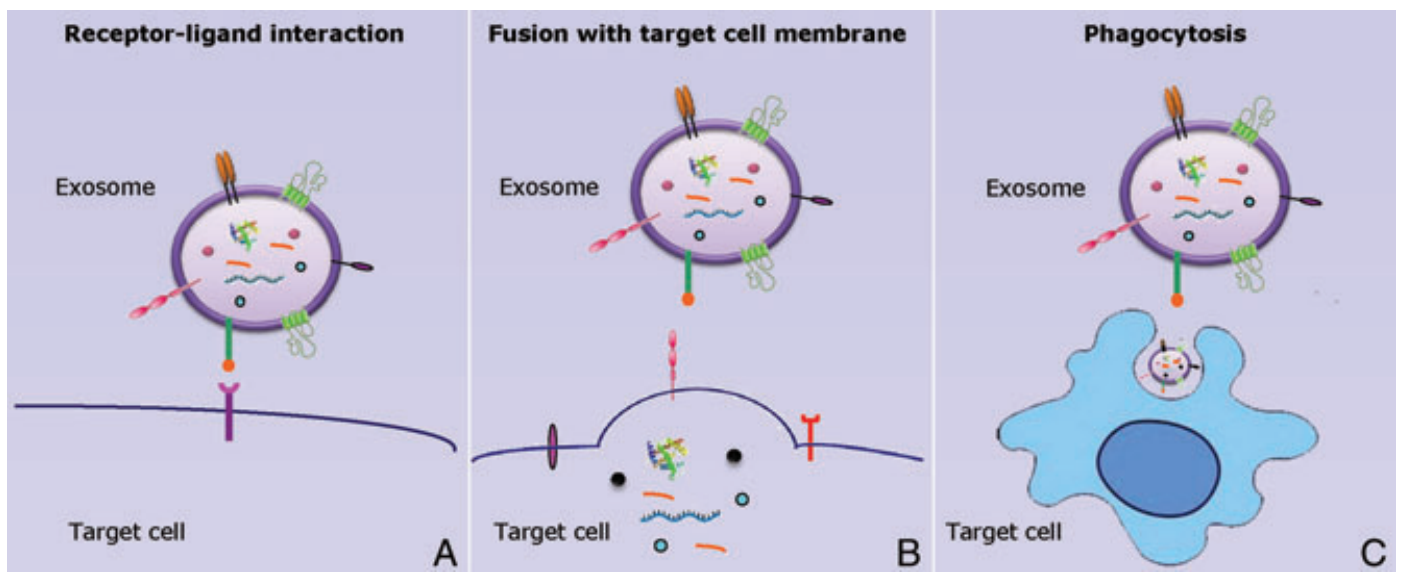

Figure 1. Mechanisms of intercellular communication mediated by exosomes. (A) Exosomes can bind to cells through receptor-ligand interactions and activate intracellular signaling. (B) Exosomes can fuse with the target cell membrane and deliver exosomal membrane and cytoplasm proteins to the recipient cell. The plasma membrane of the target cells can be also modified by the addition of new membrane receptors and different lipid components. Exosomal molecules (proteins, mRNAs, and miRNAs) can activate a multitude of signaling events in the recipient target cell. (C) An alternative exosome-cell interaction mechanism is represented by phagocytosis.

proteins [9,10]. Multivesicular bodies can fuse with lysosomes to degrade their content or with the plasma membrane to release the ILVs into the extracellular space. These vesicles, referred to as exosomes, display the endocytosed transmembrane proteins in the same orientation as those in the plasma membrane [11]. According to their origin, exosomes contain endocytic markers, i.e. tetraspanins and HSPs (as Hsc70 and Hsp90).

Initially, exosomes were described as vesicles released by reticulocytes, and they were thought to function as a way to remove unnecessary proteins, such as the transferrin receptor, during the maturation process into erythrocytes [12]. However, emerging evidence indicates exosomes as cell free messengers that play, within a tissue microenvironment, a relevant role in cell-cell communication [13]. Thus, today they are seen as novel mediators of intercellular signaling that act independently but synergistically with soluble growth factors [14]. How exosomes may interact with a target cell is not yet fully known and several mechanisms have been hypothesized (Fig. 1) [15-17]. As shown in Fig. 1A, exosomal membrane proteins can interact with the target cell in a juxtacrine fashion, acting as ligands for receptors on the cell surface. Additionally, exosomes can also fuse with the target cell resulting in the nonselective transfer of proteins and RNA from the exosome to the target cell (Fig. 1B). Finally, in a recent paper Feng et al. showed that in phagocytic cells, exosomes can be internalized efficiently via phagocytosis [15]. Their data demonstrated that exosomes promptly adhered to the cell surface of nonphagocytic cells but were not internalized, as they could be experimentally removed by trypsinization or with extensive acid washing. In contrast, the same treatment did not remove exosomes from phagocytes [15].

As result of each of these interaction mechanisms, exosomes are able to modulate selected cellular activities and participate in the signaling events regulating both physiological and pathological processes [2].

In the last decade, a large number of studies have shown that tumor cells constitutively release exosomes [18]. Their detection in supernatants of cancer cells in culture as well as in plasma, sera, and other body fluids of cancer patients has been widely reported [19-21] and all available data strongly support the protumorigenic role of tumor-derived exosomes (TDEs). Currently, MS-based proteomic technologies represent one of most powerful tools for an in-depth characterization of TDEs, providing a global view of their protein content. The present review is focused on two related aspects: (i) to provide an overview of the most characterized exosome-mediated mechanisms contributing to the pathogenesis of cancer (Fig. 2); (ii) to review recent proteomic data that support the protumorigenic role of TDEs.

\section{Role of TDEs in shaping the tumor microenvironment}

Cancer cells begin to mold their stromal environment starting with the early phases of the neoplastic process. Today, it is known that in addition to pathways involving cell-to-cell contact and the release of soluble factors, such as TNF $\alpha$, TGF $\beta$, VEGF, cancer cells are able to communicate with other cells of a tumor microenvironment (e.g. myeloid cells, fibroblasts) through the intercellular exchange of proteins and genetic materials via exosomes [22].

One of the first interesting clues concerning the understanding of TDEs' involvement in cancer was the presence of antigen-presenting complexes, such as MHC class I and II, and tumor antigens such as MelanA/Mart-1 and GP100, the carcinoembryogenic antigen (CEA), and the human epidermal growth factor receptor-2 (HER2) [23-26]. These 


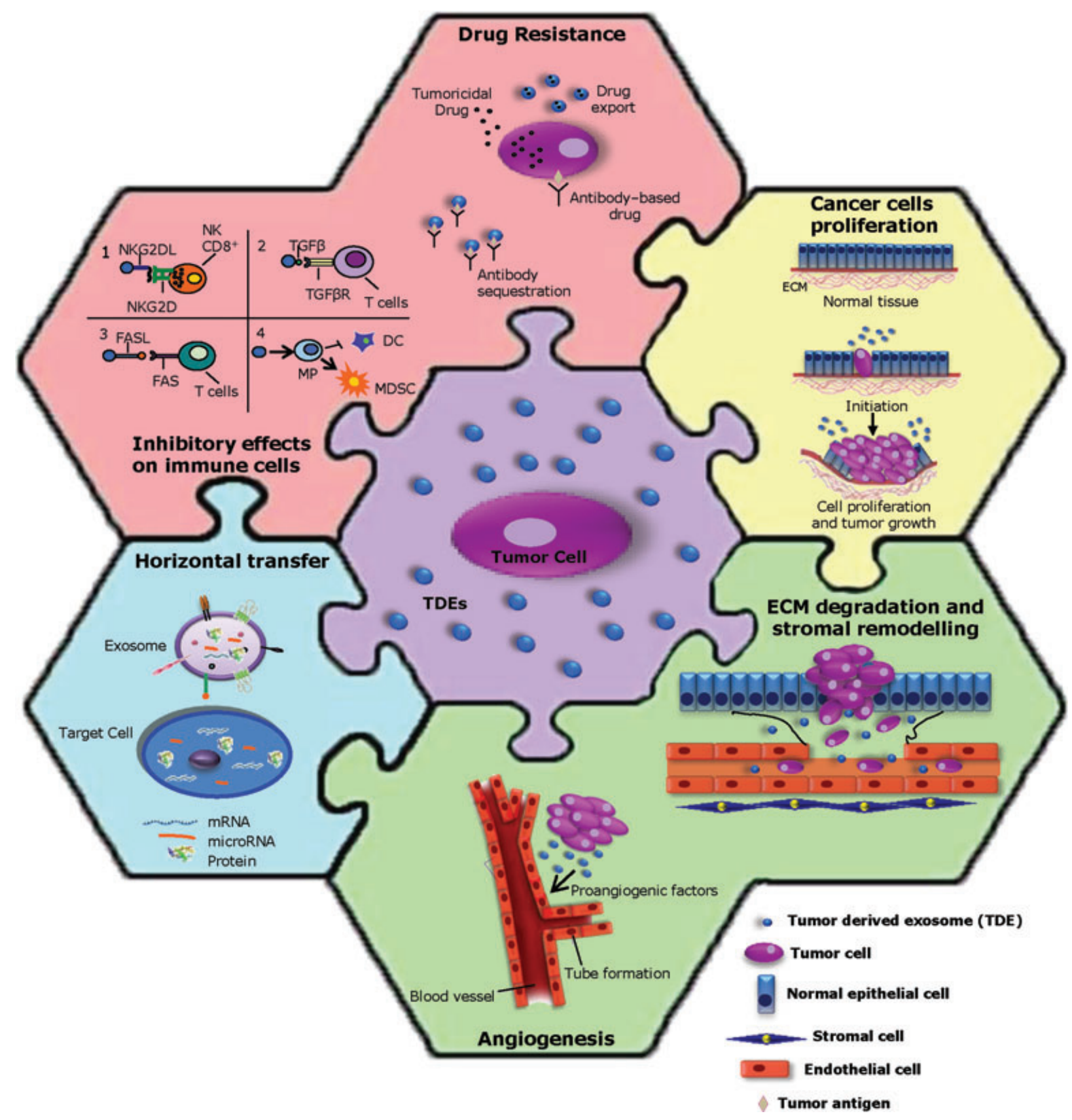

Figure 2. Pleiotropic mechanisms supporting the protumorigenic role of TDEs. TDEs modulate neoplastic cell growth via autocrine stimulation and are also able to alter the premetastatic niche by promoting angiogenesis, extracellular matrix degradation, and stromal remodeling. They also create an immunosuppressive microenvironment by impairing the cytotoxic function of NK and CD $8^{+}$cells $(1$ and 2$)$, by inducing the apoptosis of T cells through FAS-FASL ligation (3) and by altering the monocyte differentiation into dendritic cells and leading to the generation of myeloid suppressive cells (4). Furthermore, TDEs interfere with the action of therapeutic agents inducing drug resistance and are implicated in transferring miRNAs and mRNAs to neighboring cells inducing gene expression modifications.

observations have led to the hypothesis that TDEs could have an immunogenic ability to induce an effective antitumor immune response, suggesting the use of exosomes as a new delivery system for tumor antigens in cancer immunotherapy [27]. However, despite these features, immunostimulatory and antitumor effects of TDEs are rarely observed in tumors. Instead, most of the experimental data support the hypothesis that TDEs play a role in preventing antitumor immune responses and promoting tumorigenesis $[28,29]$. These effects are essentially due to the ability of TDEs (i) to induce a positive effect on neoplastic cell growth and survival, (ii) to contribute to the establishment of a premetastatic niche by 
promoting angiogenesis, extracellular matrix (ECM) degradation, and stromal remodeling, (iii) to have immunosuppressive effects, (iv) to interfere with the action of therapeutic agents by inducing drug resistance [28-32]. TDEs are also involved in transferring microRNAs (miRNAs) and mRNAs to neighboring cells, thereby inducing gene expression modulation [10,33]. A schematic representation of these pleiotropic mechanisms that support the protumorigenic effects of TDEs is reported in Fig. 2.

\subsection{TDEs induce tumor cells growth and survival}

Several studies have reported that TDEs play a critical role in the autocrine stimulation of cancer cells adding their effects to the protumoral action of soluble factors (Fig. 2). One of the first evidence supporting this role showed that the intercellular transfer of the oncogenic receptor EGFRvIII, mediated by tumor exosomes to glioma cells lacking this receptor, contributed to their morphological transformation and anchorage-independent growth [34]. Furthermore, TDEs transport apoptosis inhibitory proteins, such as survivin, a member of the apoptosis inhibitor protein family [35], they also present TGF- $\beta$ on their surface in association with betaglycan. Exosomal delivery of TGF- $\beta$ is capable of driving the differentiation of fibroblasts into myofibroblasts, whose enrichment in solid tumors provides an altered stroma that supports tumor growth, vascularization, and metastasis [36].

\subsection{Effects of TDEs in promoting the premetastatic niche}

Metastasis formation is a complex process that depends on the ability of tumor cells to induce extensive modifications in their microenvironment including (i) the degradation and remodeling of the ECM; (ii) the formation of new blood vessels (Fig. 2). TDEs are able to actively participate in shaping a premetastatic niche since they transport several biologically active molecules that play relevant roles in these processes. ECM metalloproteinase inducers, matrix metalloproteinases (MMPs), VEGF, and integrins have been found in exosomes [37-39]. Giusti et al. have also demonstrated that exosomes, derived from cells established from the ascitic fluid of a patient with ovarian cancer, show Cathepsin $\mathrm{B}$, a cystein protease, in their membrane which has a dual effect on the ECM remodeling [40]. Other exosomal proteins that have a positive effect on the invasive behavior of cancer cells are tetraspanins, cell-surface proteins spanning four times the plasma membrane [41]. They form both homocomplexes and heterocomplexes by interacting with a large variety of transmembrane and cytosolic proteins [42]. Tetraspanins promote cell spreading, cell migration, and cable formation by regulating integrin compartmentalization, internalization, recycling, and signaling $[43,44]$. In addition to enhancing the invasiveness of cancer cells, TDEs contribute to the establish- ment of a metastatic niche via the delivery of proteins, mRNAs and miRNAs that support angiogenesis. Tetraspanins themselves have been found to contribute to exosome-mediated angiogenesis by stimulating matrix metalloproteinase secretion and VEGF expression [45] in target cells. TDEs also contain several cytokines and growth factors, such as the tumor necrosis factors TNF- $\alpha$, IL- $1 \beta$, and TGF- $\beta$ as well as functional receptors such as TNFR1 that enhance the recruitment of endothelial precursor cells thus promoting neoangiogenesis [46]. Hood et al. showed that melanoma-derived exosomes stimulated endothelial cells by paracrine signaling, inducing matrix remodeling and angiogenesis [47]. Chronic myelogenous leukemia (CML) cells were also shown to release exosomes that affect in vitro and in vivo angiogenesis by inducing the expression of interleukin-8 in endothelial cells. Moreover, it was reported that HUVEC cells treated with CML exosomes increased the expression of cell-cell adhesion molecules such as VCAM-1 and ICAM-1, enhancing their ability to establish cell-cell interaction with CML cells [48].

\subsection{Immunosuppressive effects of TDEs}

Although the immune system can initially restrict tumor progression, its action is progressively blocked by the activation of suppressive pathways [49] involving cell-cell contact and the release of soluble factors [50]. Recently, mounting evidence has been pointing to TDEs as major participants in the immune evasion process (Fig. 2).

It was shown that melanoma-derived exosomes containing Fas ligand can cross-link Fas-positive T cells and trigger their apoptotic death [51] (Fig. 2). Liu et al. have also reported a death-independent effect of TDEs on the immune system. They showed that the pretreatment of mice with exosomes produced by mammary tumor cells accelerated the growth of implanted tumor cells. This effect was related to ability of TDEs to block IL-2-mediated activation of natural killer (NK) cells [52]. Moreover, it was demonstrated that the TDEs containing NKG2D ligand (such as MICA*008 or ULBP3 proteins) or membrane-bound TGF $\beta 1$, triggered the downregulation of NKG2D (an activating receptor for NK, NKT, CD8(+), and gamma-delta(+) T cells) from the surface of NK cells and CD8(+) T cells. This rapid and sustained exosomemediated decrease (Fig. 2) induced a poor functional immune response and facilitated tumor immune evasion [53,54].

Another immune escape mechanism mediated by TDEs is the induction of myeloid suppressive cells proliferation (MDSC). These are immature myeloid cells that in cancer patients are found in large number in their lymphoid organs, blood, and tumor tissues. They spontaneously secret TGF $\beta$ and inhibit T-cell proliferation and cytolytic functions. It was shown that melanoma- and colorectal carcinomaderived exosomes altered the monocyte differentiation into dendritic cells, leading to the generation of myeloid suppressive cells [21] (Fig. 2). Furthermore, it was demonstrated that the MDSC-mediated promotion of tumor progression was 
dependent on both TGF- $\beta$ and the lipidic mediator prostaglandin E2 (PGE2) transported by tumor exosomes [55].

\subsection{TDEs and drug resistance}

TDEs may indirectly contribute to tumor progression and metastasis development by interfering with the action of therapeutic agents. It was demonstrated that cisplatin-resistant ovarian cancer cells, as well as refractory melanoma cancer cells, actively expelled anticancer drugs by enhancing the release of exosomes [31, 56] (Fig. 2). Moreover, Safaei et al. reported that exosomes released by cisplatin-resistant cells were enriched in cisplatinum and expressed higher levels of the transporters MRP2, ATP7A, and ATP7B in comparison to sensitive cells [31]. Further studies showed that in ovarian cancer cells, resistance to cisplatinum is also associated with an increased secretion of annexin A3, a member of the $\mathrm{Ca}^{2+}$ and phospholipid-binding annexin family, that prevents the uptake or accumulation of platinum in cells [57]. Electron microscopy observations have shown that annexin A3 detected in culture medium was localized in exosomes, revealing yet another exosome-mediated mechanism that affects a drug's action [58].

Recently, Battke et al. have shown that exosomes can hamper the action of anticancer therapies by interfering with antibody-based drugs. The authors demonstrated that breast cancer cell lines overexpressing HER2, release exosomes expressing a full-length HER2 molecule that is able to bind, both in vitro and in vivo, to the HER2 antibody Trastuzumab resulting in a reduced amount of antibodies available for the antibody-dependent cytotoxicity (Fig. 2) [32].

\subsection{TDEs-mediated horizontal transfer of RNAs}

Recent findings have shown that exosomes contain both mRNA, and miRNAs molecules that can be delivered to recipient cells where they properly function [59] (Fig. 2). Valadi et al. demonstrated that after the transfer of mouse exosomal RNAs to human mast cells, new mouse proteins were found in the target cells, indicating that transferred exosomal mRNAs can be translated. Similarly, it has been demonstrated that exosomes also transfer miRNAs into target cells, thus regulating gene expression by their canonical binding to the target messengers $[10,33]$. The horizontal transfer of RNAs mediated by TDEs has several consequences in the regulation of tumor progression and metastasis [33,60,61]. A specific role in cancer-to-endothelial cell communication was recently reported by Umezu et al. They showed that miR-1792 cluster, especially miR-92a, contained in leukemia cells (K562) exosomes can be transferred to HUVEC endothelial cells where it significantly reduces the expression of integrin $\alpha 5$ (a target gene for miR-92a). Data obtained in this study demonstrated that K562 exosomes did not affect the growth of
HUVECs, but selectively increased endothelial cell migration and tube formation [61].

In a recent work, Fabbri et al. have showed that miR-21 and $-29 \mathrm{a}$, secreted by lung cancer cells via exosomes, can also function by acting as ligands to the human Toll-like receptor 8 (TLR8) in immune cells and by triggering a TLRmediated prometastatic inflammatory response. Thus, by acting as paracrine agonists of TLRs, these secreted miRNAs are able to regulate tumor growth and spread, working as key regulators of the tumor microenvironment [60].

\section{Contribution of proteomics study in defining the protumoral role of TDEs}

In the last years the increased application of proteomic technologies has significantly contributed to deeply delineate the protein profile of exosomes from a variety of cultured cells and body fluids (such as plasma, urine, and tumor effusions) [62-66]. A part of these studies are specifically focused on TDEs and the data are providing new insights for understanding their role in the regulation of tumor microenvironment [62-65].

\subsection{Technical features of TDE proteomics}

In the field of exosome proteomics, the purification and characterization of nanovesicles are the two most critical points to control in order to avoid false-positive identifications due to the presence of contaminating components [67]. Factors such as biological matrices (e.g. cell culture media or biological fluids) must be carefully considered in the choice of suitable methods for exosome purification allowing the elimination of the abundant nonexosomal proteins including Igs and albumin. The most used isolation strategies involve a combination of different methods such as: differential centrifugation, filtration, concentration, flotation density gradient, and immunocapture beads. Moreover, after a first isolation step, an appropriate characterization by transmission electron microscopy, fluorescent activated cell-sorted analysis, and/or western blot is suggested to validate the relevance of the recovered material $[2,67]$.

However, despite the efforts to improve the purification of exosomes, the methods that are typically used today are still not completely able to guarantee the absence of nonexosomal contaminant proteins and for this reason the strategies used to purify exosomes often differ between laboratories with little consensus concerning their criteria of purity. Nevertheless, during the last few years the advances in proteomic technology have facilitated the collection of extensive exosome protein data sets. Among the about 80 papers on exosome proteomics (including reviews) published during the last 80 years, less than 20 are on TDEs. Moreover, most of them describe expression proteomic studies focused on defining exosome proteome profiling and very often are 


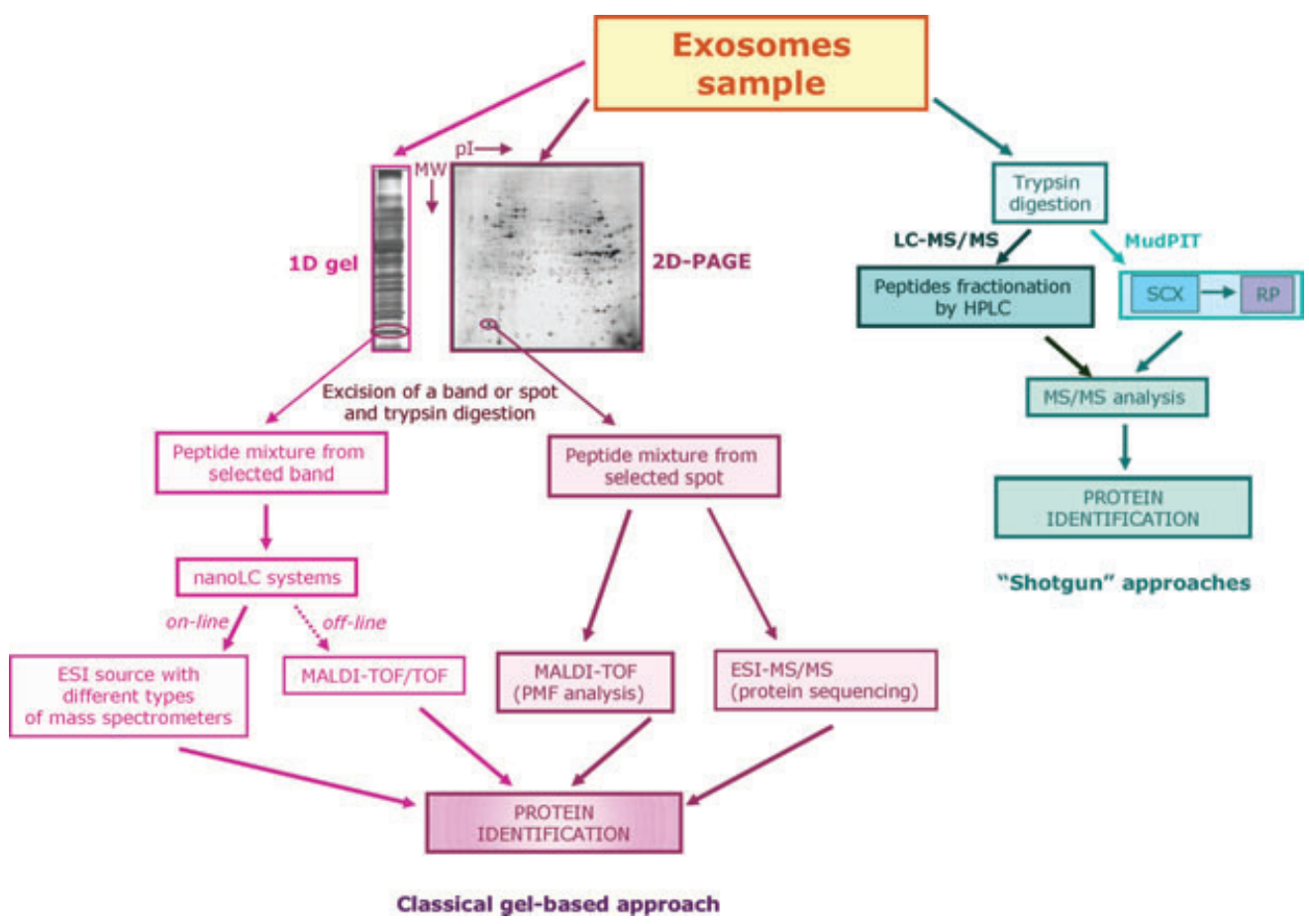

Figure 3. Overview of methodological approaches in proteomic exosome research. Exosome proteins may be separated by 1- or 2DE and proteins contained in individual bands or spots, after "in gel" trypsin digestion, are identified by MALDI-TOF/TOF or ESI-MS/MS (classical gel-based approach). Alternatively, an exosome sample can be processed by performing a shotgun proteomic approach such as MudPIT. This technique consists of a 2D chromatography separation of peptides generated by the enzymatic digestion of sample, followed by MS/MS analysis on LTQ or Orbitrap. SCX: strong cation exchange.

specifically orientated toward the discovery of tumor biomarkers $[25,62,63,65,68]$.

Currently, two strategies are mostly used to define an exosome protein content: the first analytical approach is based on gel electrophoresis separation followed by MS analysis; the second one is based on the application of several shotgun proteomics procedures such as LC-MS/MS or multidimensional protein identification technology (MudPIT) [69] (Fig. 3).

In the former approach, proteins are first separated by SDS-PAGE (1D or 2DE) and gel bands or spots are then cut, trypsin-digested, and analyzed by MS. Peptide mixtures derived from 2D gel spots that can be analyzed by PMF, performing MALDI-TOF MS or by peptide sequencing, performing an MS/MS $[69,70]$. For the identification of proteins separated by $1 \mathrm{D}$ gel, since a single gel band may contain more than one protein, the classical approach based on PMF cannot be used. Therefore, tryptic peptides must be separated by nanoLC systems online connected through an ESI source with different types of mass spectrometers $[16,71]$ or analyzed off-line by MALDI-TOF/TOF [65].

Alternatively, for the proteomic profiling of exosomes, gelfree methods such as LC-MS/MS or MudPIT have been used. The LC-MS/MS method allows for the concentration and isolation of hundreds of selected peptides from extremely complex mixtures obtained through the proteolytic digestion of proteins, before sequencing by MS $[63,72]$. In MudPIT analysis, peptides derived from digested proteins are first separated with a strong cation exchange column followed by a RP separation online connected to LTQ or Orbitrap mass spectrometer, thus combining the separation steps with the identification process [73].

\subsection{Proteomics data set of TDEs}

In general, the available published data on TDE proteomics clearly shows that proteins identified in these nanovesicles (both released by tumor cell lines and isolated from body fluids) can be sorted into two groups: one group represents a conserved set of proteins irrespective of exosome origin; the second one is formed by proteins specifically related to the producer host cell, showing that TDEs have a unique cell-specific protein composition.

Within the group of common proteins, those most frequently identified belong to the following classes: membrane adhesion proteins (integrins); components of the ESCRT machinery (alix, TSG101, vacuolar protein sorting-associated protein 28 homolog (vps-28), vacuolar protein sorting-associated protein 4B (vps-4B), ubiquitinlike modifier-activating enzyme, and ubiquitin); membrane transport/trafficking (annexins, Rab protein family); 
cytoskeletal components (actin, cytokeratins, ezrin, tubulin, and myosin); lysosomal markers (lysosome membrane protein 2, cathepsin-D, CD63, LAMP-1/2); antigen presentation proteins (HLA class I and II/peptide complexes); metabolic enzymes (GAPDH, pyruvate, enolase alpha); HSPs (Hsc70, Hsp70, Hsp90); kinases (LYN, MINK1, and MAP4K4); tetraspanins (CD9, CD81, CD82, tetraspanin-8) proteases (ADAM10, DPEP1, ST14); transporters (ATP7A, ATP7B, MRP2, SLC1A4, SLC16A1, CLIC1); receptors (CD46, CD55, NOTCH1) $[16,26,65,70,74]$. Many of these proteins have been cataloged in the ExoCarta website (http://www.exocarta.org/).

As more proteome studies are performed, it is becoming ever more apparent that beyond the set of conserved proteins, TDEs contain proteins that are not found in the exosomes from both nontumor cells and/or body fluids of healthy individuals $[16,25,26,62,63,65,74,75]$. All of the proteomics data that have been obtained so far demonstrates that TDEs express a discrete set of proteins specifically related to the tumor phenotype and involved in cell proliferation, antigen presentation, signal transduction, migration, invasion, and angiogenesis, supporting the hypothesis that exosomes may play a crucial role in modulating tumor progression and preparing the metastatic niche.

A large amount of proteomic data regarding TDEs has been obtained from studies on human colorectal cancer (CRC) cell-derived exosomes. In the first global proteomic analysis performed on exosomes released from HT29 CRC cells, a total of 547 proteins were identified and among them 181 had never been previously described as exosome proteins. Most of these proteins were involved in exosome biogenesis, but a part of them were related to processes related to tumorigenesis [75]. In particular, this subset of 28 proteins have a role in cancer development, including the ADAM10, 14-3-3 proteins, CD44, CD82, CD9, Alix (PDC6I), Ras-related proteins, syntenin-1, MIF, and tetraspanin 8 , which were already described in other cell-derived exosomes, as well as the newly reported proteins in exosomes such as $\beta$-catenin, RACK1, chondroitin sulfate proteoglycan NG2, E-cadherin, and ephrin B1. All of them contribute to the tumor phenotype and are differentially involved in the regulation of tumor progression via the promotion of tumor growth, migration, invasion, immune modulation, metastasis, and angiogenesis [75] (Table 1).

More recently, a comparative proteomic analysis of LIM1215 (colorectal cancer)-derived exosomes with human urine- and murine mast cell-derived exosomes showed that among the 394 identified proteins, 250 were exclusively present in the LIM1215 exosomes, revealing a tissueassociated protein signature. Among them, a subset of proteins known to be expressed in colorectal cancer tissues was also found. This set included A33 antigen, cadherin-17, CEA, epithelial cell surface antigen (EpCAM), proliferating cell nuclear antigen, epidermal growth factor receptor, mucin 13, misshapen-like kinase 1, mitogen-activated protein kinase 4, claudins (1, 3, and 7), centrosomal protein $55 \mathrm{kDa}$, ephrin$\mathrm{B} 1$ and -B2, and the enzyme phospholipid scramblase [16]
(Table 1). Interestingly, this study also revealed that the LIM1215 exosome proteins could be categorized in some of the seven cancer hallmark features $[76,77]$. In particular, $36 \%$ belong to the category of self-sufficiency in growth signals, $24 \%$ had a role in limitless replicative potential, $17 \%$ of the proteins were upregulated in colon cancer metastasis, whereas $6 \%$ function in apoptosis evasion [16].

Similar results were reported by Tauro and colleagues in a study focused on the proteomic characterization of exosomes derived from another colon carcinoma cell line (LIM1863). They found that among the 171 unique proteins identified in these exosomes, many were associated with cancer cell proliferation, invasion, and metastasis such as c-Met protein (hepatocyte growth factor receptor), amphiregulin (AREG), various ephrins (EFNB1, EFNB2), and Eph receptors (EPHA2-8, EPHB1-4), components involved in Wnt ( $\beta$ Catenin and TNIK) and Ras (CRK and GRB2) signaling, and several key components associated with colorectal cancer, including CEACAM1 and 5, FAT1, and CDH17 [74]. It is a well-known fact that CEACAM5 (CEA) is found to be overexpressed in over $90 \%$ of human gastrointestinal and pancreatic cancers and, in particular it is associated with metastatic potential in colon cancer $[78,79]$ (Table 1 ).

Additional information relative to the protein composition of CRC exosomes was provided from the proteome profiling of highly purified exosomes derived from human CRC ascites [63]. In this study, together with proteins related to tumor progression and already identified in previous studies $[16,75]$, the authors reported the presence of new proteases such as aminopeptidase $\mathrm{N}$, angiotensin-converting enzyme, which play important roles in tumor invasion, metastasis, and angiogenesis as well as several proteins that affect epithelial cellular integrity and polarity (as galectins, Crumbs homolog 2, adherens junction and tight-junction proteins, and claudins) [63] (Table 1).

Other interesting data supporting the role of TDEs in tumor biology was produced by proteomics studies carried out on other types of human tumors, such as breast cancer [70], bladder cancer [65], mesothelioma [25], melanoma [26], and medulloblastoma [80], but also from murine insulinoma [71]. For example, the statistically based, unbiased overrepresentation analysis of human bladder cancer exosomes highlighted that these exosomes expressed proteins strongly related to neoplastic diseases in general and to carcinomas in particular.

Moreover, it was demonstrated that in the biological processes category, a large proportion of identified proteins had functional roles in the control of the cytoskeleton, intercellular adhesion, matrix adhesion processes, and protein foldingrelated processes [65]. All of these data clearly demonstrates that TDEs are characterized by a proteome profile strongly related to their origin and determining their functions.

Table 1 shows a summary of proteins identified in TDEs known for their role in regulating several steps of cancer progression. 
Table 1. Exosome proteins associated with tumor progression $[16,25,26,63,65,70,71,74,75,80]$

\begin{tabular}{|c|c|c|c|}
\hline Protein name & $\begin{array}{l}\text { Uniprot accession } \\
\text { number }^{\text {a) }}\end{array}$ & $\begin{array}{l}\text { Role in tumor } \\
\text { progression }\end{array}$ & $\begin{array}{l}\text { Tumor-exosome } \\
\text { origin }\end{array}$ \\
\hline 14-3-3 protein beta/alpha & P31946 (H) Q9CoV8 (M) & Tumor growth & CRC, BIC, Ins \\
\hline 14-3-3 protein epsilon & P62258 (H) P62259 (M) & Tumor growth & $\mathrm{CRC}, \mathrm{BC}, \mathrm{BIC}$, Ins \\
\hline 14-3-3 protein eta & Q04917 (H) P68510 (M) & Tumor growth & CRC, Ins \\
\hline 14-3-3 protein gamma & P61981 (H) P61982 (M) & Tumor growth & CRC, BC, BIC, Ins \\
\hline $14-3-3$ protein theta & P27348 (H) P68254 (M) & Tumor growth & $\mathrm{CRC}, \mathrm{BC}, \mathrm{BIC}$, Ins \\
\hline 14-3-3 protein sigma & P31947 & Tumor growth, metastasis & $\mathrm{BIC}$ \\
\hline 14-3-3 protein zeta/delta & P63104 (H) P63101 (M) & Tumor growth & $\mathrm{CRC}, \mathrm{BC}, \mathrm{BIC}$, Ins \\
\hline ADAM 10 & 014672 & $\begin{array}{l}\text { Tumor growth, migration, invasion, } \\
\text { angiogenesis }\end{array}$ & CRC \\
\hline Adapter molecule crk & P46108 & Migration, invasion & CRC \\
\hline $\begin{array}{l}\text { Alix (programmed cell death } \\
6 \text {-interacting protein-PDC6I) }\end{array}$ & Q8WUM4 & Tumor growth & $\mathrm{CRC}, \mathrm{BC}, \mathrm{BIC}$ \\
\hline Aminopeptidase N (CD13) & P15144 & Migration, angiogenesis & CRC \\
\hline Amphiregulin & P15514 & Tumor growth & CRC \\
\hline Angiotensin-converting enzyme & P12821 & Invasion, angiogenesis & CRC \\
\hline Annexin A1 & P04083 & Tumor growth, invasion & CRC, BC, BIC, Mes \\
\hline Annexin A2 & P07355 & Tumor growth, invasion & $\begin{array}{l}\text { CRC, BC, BIC, Mes, } \\
\text { Mel, MedBI }\end{array}$ \\
\hline Annexin A3 & P12429 & Tumor growth, drug resistance & $\mathrm{CRC}$ \\
\hline Annexin A4 & P09525 & Tumor growth, drug resistance & $\mathrm{CRC}, \mathrm{BC}, \mathrm{BIC}$ \\
\hline Annexin A5 & P08758 (H) P48036 (M) & Tumor growth, invasion & $\begin{array}{l}\text { CRC, BC, BIC, Ins, } \\
\text { Mes, Mel }\end{array}$ \\
\hline Annexin A6 & P08133 & Invasion & $\begin{array}{l}\text { CRC, BC, BIC, Mes, } \\
\quad \text { Mel }\end{array}$ \\
\hline Annexin A7 & P20073 & Tumor growth, migration, invasion & $\mathrm{BIC}$ \\
\hline Beta-2-microglobulin & P61769 & Immune modulation & $\mathrm{CRC}, \mathrm{BC}, \mathrm{BIC}$ \\
\hline $\begin{array}{l}\text { Carcinoembryonic } \\
\text { antigen-related cell adhesion } \\
\text { molecule } 5 \text { precursor (CEA) }\end{array}$ & P06731 & Tumor growth & $\mathrm{CRC}$ \\
\hline Basigin & P35613 & Migration, invasion & $\mathrm{CRC}, \mathrm{BIC}$ \\
\hline Cadherin-1 & P12830 & Invasion & CRC \\
\hline Cadherin-17 & Q12864 & Invasion, metastasis & $\mathrm{CRC}$ \\
\hline Catenin beta-1 & P35222 & Tumor growth & $\mathrm{CRC}, \mathrm{BIC}$ \\
\hline Catenin delta-1 & 060716 & Tumor growth & $\mathrm{BIC}, \mathrm{Mel}$ \\
\hline CD151 antigen & P48509 & Migration, invasion & $\mathrm{CRC}$ \\
\hline CD44 & P16070 & Tumor growth, invasion, angiogenesis & CRC, BIC \\
\hline CD82 antigen & P27701 & Immune modulation, invasion & $\mathrm{CRC}$ \\
\hline CD9 antigen & P21926 & Immune modulation, migration, invasion & CRC \\
\hline CD97 antigen & P48960 & Invasion & $\mathrm{CRC}$ \\
\hline $\begin{array}{l}\text { Cell division control protein } 42 \\
\text { homolog (CDC42) }\end{array}$ & P60953 & Tumor growth, invasion, metastasis & $\mathrm{CRC}, \mathrm{BIC}$ \\
\hline Cell surface $A 33$ antigen & Q99795 & $\begin{array}{l}\text { Cell-cell recognition and signaling, tumor } \\
\text { antigen }\end{array}$ & $\mathrm{CRC}$ \\
\hline Centrosomal protein of $55 \mathrm{kDa}$ & Q53EZ4 & Tumor growth & $\mathrm{CRC}$ \\
\hline $\begin{array}{l}\text { Chondroitin sulfate } \\
\text { proteoglycan NG2 }\end{array}$ & Q6UVK1 & Angiogenesis & $\mathrm{CRC}$ \\
\hline Claudin-3 & 015551 & Disruption of epithelial cell polarity, invasion & CRC \\
\hline Claudin-7 & 095471 & Disruption of epithelial cell polarity, invasion & $\mathrm{CRC}$ \\
\hline Crumbs homolog 2 & Q5IJ48 & Disruption of epithelial cell polarity, invasion & CRC \\
\hline Dipeptidyl peptidase 4 & P27487 & Invasion & $\mathrm{CRC}$ \\
\hline $\begin{array}{l}\text { DNA polymerase delta catalytic } \\
\text { subunit }\end{array}$ & P28340 & Tumor growth, invasion & CRC \\
\hline $\begin{array}{l}\text { Epidermal growth factor } \\
\text { receptor }\end{array}$ & P00533 & Tumor growth, migration & $\mathrm{CRC}, \mathrm{BIC}$ \\
\hline $\begin{array}{l}\text { Epithelial cell surface antigen } \\
\text { (EpCAM) }\end{array}$ & P16422 & Tumor growth, invasion & $\mathrm{CRC}$ \\
\hline EPH receptor B2 & B1АКС9 & Tumor growth, invasion & CRC \\
\hline EPH receptor B3 & D3DNT9 & Tumor growth, invasion, metastasis & CRC \\
\hline
\end{tabular}


Table 1. Continued

\begin{tabular}{|c|c|c|c|}
\hline Protein name & $\begin{array}{l}\text { Uniprot accession } \\
\text { number }\end{array}$ & $\begin{array}{l}\text { Role in tumor } \\
\text { progression }\end{array}$ & $\begin{array}{l}\text { Tumor-exosome } \\
\text { origin }\end{array}$ \\
\hline Ephrin B1 & P98172 & Tumor growth, invasion, metastasis & CRC \\
\hline Ephrin B2 & P52799 & Tumor growth, invasion, metastasis & $\mathrm{CRC}$ \\
\hline Ezrin & P15311 (H) P26040 (M) & Tumor growth, migration, invasion & $\begin{array}{l}\text { CRC, BC, BIC, Ins, } \\
\text { Mel, Mes, MedBI }\end{array}$ \\
\hline Fascin & Q16658 & Migration, invasion & CRC, BC, BIC, Mes \\
\hline Galectin-1 & P09382 & Angiogenesis, metastasis, drug resistance & $\mathrm{CRC}, \mathrm{BIC}$ \\
\hline Galectin-3 & P17931 & Tumor growth & $\mathrm{CRC}, \mathrm{BC}, \mathrm{BIC}$ \\
\hline Galectin-3-binding protein & Q08380 & Migration, metastasis & $\mathrm{CRC}, \mathrm{BC}, \mathrm{BIC}$ \\
\hline Galectin-4 & P56470 & Tumor growth & $\mathrm{CRC}$ \\
\hline GST Mu 1 & P10649 & Tumor growth, drug resistance & Ins \\
\hline GST omega-1 & P78417 & Tumor growth, drug resistance & $\mathrm{CRC}, \mathrm{BC}$ \\
\hline GST P & P09211 (H) P19157 (M) & Tumor growth, drug resistance & CRC, BIC, Ins \\
\hline $\begin{array}{l}\text { Growth factor receptor-bound } \\
\text { protein } 2 \text { (Adapter protein } \\
\text { GRB2) }\end{array}$ & P62993 & Tumor growth & $\mathrm{CRC}$ \\
\hline GTPase Kras & P01116 & Tumor growth & CRC \\
\hline $\begin{array}{l}\text { Guanine nucleotide-binding } \\
\text { protein subunit beta-2-like } 1 \\
\text { (RACK1) }\end{array}$ & P63244 & Tumor growth, angiogenesis, & CRC \\
\hline $\begin{array}{l}\text { Heat shock cognate } 71 \mathrm{kDa} \\
\text { protein }\end{array}$ & P11142 & Tumor growth & $\begin{array}{l}\text { CRC, BC, Mel, } \\
\text { MedBI }\end{array}$ \\
\hline HSP beta-1 & P04792 & Tumor growth & CRC, BC, MedBI \\
\hline $\begin{array}{l}\text { Hepatocyte growth factor } \\
\text { receptor (c-Met) }\end{array}$ & P08581 & Tumor growth, metastasis & CRC \\
\hline $\begin{array}{l}\text { Integrin-binding protein DEL1 } \\
\text { precursor }\end{array}$ & 043854 & Angiogenesis & Mes \\
\hline $\begin{array}{l}\text { Intercellular adhesion molecule } \\
1 \text { (ICAM-1) }\end{array}$ & P05362 & Metastasis & CRC \\
\hline Junction plakoglobin & P14923 & Invasion & CRC \\
\hline Lin-7 homolog C & Q9NUP9 & Disruption of epithelial cell polarity, invasion & CRC \\
\hline $\begin{array}{l}\text { Macrophage migration } \\
\text { inhibitory factor (MIF) }\end{array}$ & P14174 & $\begin{array}{l}\text { Tumor growth, immune modulation, invasion, } \\
\text { angiogenesis }\end{array}$ & CRC \\
\hline Moesin & P26038 & Tumor growth, migration, invasion & CRC, BIC, Mes, Mel \\
\hline Mucin-1 & P15941 & Tumor growth & CRC, BIC \\
\hline Mucin-13 & Q9H3R2 & Tumor growth & $\mathrm{CRC}$ \\
\hline Mucin-16 & B5ME49 & Tumor growth & CRC \\
\hline Misshapen-like kinase 1 & Q8N4C8 & Tumor growth, migration & CRC \\
\hline $\begin{array}{l}\text { Mitogen-activated protein } \\
\text { kinase } 4\end{array}$ & 095819 & Tumor growth & $\mathrm{CRC}$ \\
\hline Olfactomedin 4 & Q6UX06 & Tumor growth & $\mathrm{CRC}$ \\
\hline Peroxiredoxin-1 & $\mathrm{Q} 06830(\mathrm{H}) \mathrm{P} 35700(\mathrm{M})$ & Tumor growth & CRC, BC, BIC, Ins \\
\hline Peroxiredoxin-2 & P32119 (H) Q61171 (M) & Tumor growth & $\mathrm{CRC}, \mathrm{BC}$, Ins \\
\hline Peroxiredoxin-3 & P30048 & Tumor growth & $\mathrm{CRC}, \mathrm{BC}$ \\
\hline Peroxiredoxin-4 & Q13162 (H) O08807 (M) & Tumor growth & $\mathrm{BC}$, Ins \\
\hline Peroxiredoxin-5, mitochondrial & P30044 & Tumor growth & $\mathrm{CRC}, \mathrm{BIC}$ \\
\hline Peroxiredoxin-6 & P30041 & Invasion, metastasis & $\mathrm{CRC}, \mathrm{BC}, \mathrm{BIC}$ \\
\hline $\begin{array}{l}\text { Phosphatidylethanolamine- } \\
\text { binding protein } 1\end{array}$ & P30086 & Tumor growth & $\mathrm{CRC}$ \\
\hline Phospholipid scramblase 1 & 015162 & Tumor growth & CRC \\
\hline Plexin B2 & 015031 & Migration, invasion, angiogenesis & CRC \\
\hline $\begin{array}{l}\text { Proliferating cell nuclear } \\
\text { antigen }\end{array}$ & P12004 & Tumor growth & CRC \\
\hline $\begin{array}{l}\text { Prostaglandin F2 receptor } \\
\text { negative regulator }\end{array}$ & Q9P2B2 & Migration, invasion & $\mathrm{CRC}, \mathrm{BIC}$ \\
\hline Protocadherin Fat 1 & Q14517 & Migration, invasion & CRC \\
\hline Radixin & P35241 & Tumor growth, migration, invasion & $\begin{array}{l}\text { CRC, BIC, Mel, } \\
\text { MedBI }\end{array}$ \\
\hline
\end{tabular}


Table 1. Continued

\begin{tabular}{|c|c|c|c|}
\hline Protein name & $\begin{array}{l}\text { Uniprot accession } \\
\text { number }^{\text {a) }}\end{array}$ & $\begin{array}{l}\text { Role in tumor } \\
\text { progression }\end{array}$ & $\begin{array}{l}\text { Tumor-exosome } \\
\text { origin }\end{array}$ \\
\hline Ras-related protein R-Ras & P10301 & Invasion & $\mathrm{CRC}$ \\
\hline Ras-related protein R-Ras2 & P62070 & Invasion & CRC \\
\hline $\begin{array}{l}\text { Receptor tyrosine-protein } \\
\text { kinase erbB-2 }\end{array}$ & P04626 & Tumor growth & MedBI \\
\hline RuvB-like 1 & Q9Y265 & Tumor growth & $\mathrm{CRC}$ \\
\hline $\begin{array}{l}\text { Serine/threonine-protein } \\
\text { phosphatase } 2 \mathrm{~A} 65 \mathrm{kDa} \\
\text { regulatory subunit } \mathrm{A} \text { beta } \\
\text { isoform }\end{array}$ & P62715 & Tumor growth & Ins \\
\hline $\begin{array}{l}\text { Serine/threonine-protein } \\
\text { phosphatase } 2 \mathrm{~A} 65 \mathrm{kDa} \\
\text { regulatory subunit } \mathrm{A} \text { beta } \\
\text { isoform }\end{array}$ & P30154 (H) Q76MZ3 (M) & Tumor growth & CRC, Ins \\
\hline Syntenin-1 & 000560 & Invasion & CRC, mel \\
\hline Tetraspanin-1 & 060635 & Tumor growth, migration, invasion & CRC \\
\hline Tetraspanin-8 & P19075 & Angiogenesis & $\mathrm{CRC}$ \\
\hline TNIK protein & Q7Z4L4 & Tumor growth & CRC \\
\hline Transgelin-2 & P37802 (H) Q9WVA4 (M) & Tumor growth & CRC, BC, BIC, Ins \\
\hline Trophoblast glycoprotein & Q13641 & Migration & $\mathrm{CRC}$ \\
\hline Tyrosine-protein kinase Lyn & P07948 & Tumor growth, migration & $\mathrm{CRC}$ \\
\hline Vinculin & P18206 & Tumor growth, invasion & $\mathrm{CRC}, \mathrm{BC}$ \\
\hline
\end{tabular}

CRC: colorectal cancer; BC: breast cancer; BIC: bladder cancer; Ins: insulinoma; Mes: mesothelioma; Mel: melanoma; MedBI: medulloblastoma.

a) $\mathrm{H}$ : human protein; $\mathrm{M}$ : mouse protein. Two accession numbers are reported for proteins identified in both human (CRC, BC, BIC, Mes, Mel, MedBI) and murine cancers (Ins).

\subsection{The new challenge of TDE proteomics}

Most of the proteomic studies performed on TDEs up to now have allowed for the catalogization of the proteins that they contain. However, although TDEs' proteins have been also classified for their biological functions, from a Systems Biology perspective the large-scale nature of TDE proteomics data has been so far largely untapped.

Proteomics experimental results, beyond a rapid identification of a high number of proteins in a single analysis, provide distinct data types that can be used to quantify proteins between different proteome states, including the temporal variation of the proteome, to determine the complete primary structure of proteins including PTMs, and to determine protein interactions [81]. These aspects of expression and functional proteomics could provide new knowledge for understanding the mechanisms of protein sorting into TDEs, the biological meaning of some proteins in TDEs, and the interrelationships between TDE proteins.

Few papers, all published during the last 3 years, have reported data concerning quantitative and PTMs analysis of TDE proteins, as well as data depicting protein-protein interaction networks in TDEs.

In a work performed on breast cancer exosomes, a quantitative and comparative proteomic analysis was carried out between exosomes and whole cell lysate. This analysis revealed that vesicles had a distinctive protein profile in comparison to the producing cell. The functional clustering of the enriched exosomal proteins showed that most of them belonged to functional classes of cytoskeleton, the regulation of programmed cell death and signal proteins that, as is published, are differentially involved in mechanisms affecting the malignant progression of cancer [70]. Other significant data were obtained by a quantitative proteomics study carried out on the A431 human epidermoid carcinoma. In this work, the authors reported that when under hypoxia, cells secreted higher levels of proteins involved in angiogenesis, focal adhesion formation, ECM-receptor interaction, and immune cell recruitment in comparison to cells undergoing normoxic and reoxygenation conditions. They found that more than $50 \%$ of these secreted proteins, predominantly classified as cytoplasmic and membrane proteins, were localized in exosomes [82]. Currently, new methods of performing quantitative proteome analyses based on differential labeling protocols or labelfree techniques as well as a vast number of software tools for the analysis of quantitative proteomics data are almost monthly described in the literature [69,83-85]. During the next few years, the application of these accurate quantitative techniques could represent one of most challenging issues in the field of TDE proteomics.

Although it is well known that the identification of PTMs is very often essential for understanding the real function of proteins and their localization within cells or cell organelles, only one paper in the published literature reports data concerning PTMs of exosomal proteins. In this work, Lee and co-workers analyzed exosomes derived from murine 
insulinoma cells and showed that some proteins (such as ubiquitin and HSP90ß) were present in secreted vesicles as multiple populations, distinct from those observed in cell lysate. By analyzing data obtained combining techniques such as 1D gel separation, western blot, and nanoLC-ESI-q-TOF MS/MS, the authors highlighted that these multiple forms of a same protein were due to several PMTs, including ubiquitination and phosphorylations. On the basis of these observations, they propose that some uniquely modified proteins could be located in exosomes. However, the relationship between PTMs detected in exosomes and their biogenesis or their functions was not further investigated and no more data are currently available.

Doubtless, different independent difficulties (such as low abundance, lability of the modifications) have made it challenging to perform a PTM global analysis so far. However, technological advances in MS-based proteomics have allowed some critical points to be overcome and MS-based PTM analysis is now quite successful. Good results have been produced not only for phosphorylation (the more studied PTM), but also for lysine acetylation or methylation, $\mathrm{N}$-glycosylation, ubiquitination, sumoylation, and others that have also been investigated in a quantitative manner [81] and references therein]. The possibility to characterize the PTMs in TDEs proteins as well as their functional effects, may represent an outstanding development of TDEs proteomic studies, furthering our understanding of TDE roles in tumor microenvironment.

Finally, today the characterization of protein networks is a highly valued goal of the postgenomic studies. However, this aspect remains almost completely unexplored in the field of exosome proteomics. The only data available in literature comes from two recent studies in which exosomes derived from human medulloblastoma and colorectal carcinoma cells were analyzed. Proteomic data obtained in these studies allowed the construction and analysis of proteinprotein interaction networks for TDEs for the first time, revealing that exosomal proteins are closely interconnected via physical interactions and cluster into functional modules involved in the biogenesis of exosomes and in several processes regulating tumor progression (as cell proliferation, cell migration, and immune suppression) $[80,86]$. Interestingly, what has clearly emerged from the work on CRCderived exosomes is that these TDEs have several hub proteins, with many other vesicular proteins that have few interacting partners, and well-connected clusters similar to those identified in other subcellular networks. However, there is a significant amount of evidence that indicates that groups of proteins with roles in specific biological processes and molecular functions are highly enriched or depleted in TDEs, suggesting that TDEs are extracellular organelles distinct from other intracellular organelles and compartments [86]. Further Systems Biology approaches could provide a new integrated view of TDEs, and the resulting interactome data, analyzed extensively through bioinformatic methods, would offer new tools for defining the organization and function of exosomes.
In conclusion, the involvement of TDEs in promoting tumor progression is supported by all of the proteomic data obtained from several types of tumors. However, up until now the exosome proteomic study has been often utilized to catalog TDEs' proteins in order to find disease biomarkers rather than to predict the functional activities of the vesicles. Thus, new efforts must currently focus on the development of new perspectives to perform adequate quantitative, PTMs, and protein interaction analyses with the aim of creating predictive models of TDEs in the field of Systems Biology.

This work was supported by Italian Association for Cancer Research (AIRC) to R.A.; University of Palermo (International Cooperation) to S.F.; Ministry of University and Scientific Research (ex 60\%) to R.A. and S.F.

The authors have declared no conflict of interest.

\section{References}

[1] Thery, C., Zitvogel, L., Amigorena, S., Exosomes: composition, biogenesis and function. Nat. Rev. Immunol. 2002, 2, 569-579.

[2] Simpson, R. J., Jensen, S. S., Lim, J. W., Proteomic profiling of exosomes: current perspectives. Proteomics 2008, 8 , 4083-4099.

[3] Caby, M. P., Lankar, D., Vincendeau-Scherrer, C., Raposo, G. et al., Exosomal-like vesicles are present in human blood plasma. Int. Immunol. 2005, 17, 879-887.

[4] Pisitkun, T., Shen, R. F., Knepper, M. A., Identification and proteomic profiling of exosomes in human urine. Proc. Natl. Acad. Sci. USA 2004, 101, 13368-13373.

[5] Skriner, K., Adolph, K., Jungblut, P. R., Burmester, G. R., Association of citrullinated proteins with synovial exosomes. Arthritis Rheum. 2006, 54, 3809-3814.

[6] Andre, F., Schartz, N. E., Movassagh, M., Flament, C. et al., Malignant effusions and immunogenic tumour-derived exosomes. Lancet 2002, 360, 295-305.

[7] Gatti, J. L., Metayer, S., Belghazi, M., Dacheux, F. et al., Identification, proteomic profiling, and origin of ram epididymal fluid exosome-like vesicles. Biol. Reprod. 2005, 72, 1452-1465.

[8] Ronquist, G., Brody, I., The prostasome: its secretion and function in man. Biochim. Biophys. Acta 1985, 822, 203-218.

[9] Thery, C., Boussac, M., Veron, P., Ricciardi-Castagnoli, P. et al., Proteomic analysis of dendritic cell-derived exosomes: a secreted subcellular compartment distinct from apoptotic vesicles. J. Immunol. 2001, 166, 7309-7318.

[10] Valadi, H., Ekstrom, K., Bossios, A., Sjostrand, M. et al., Exosome-mediated transfer of mRNAs and microRNAs is a novel mechanism of genetic exchange between cells. Nat. Cell Biol. 2007, 9, 654-659.

[11] Keller, S., Sanderson, M. P., Stoeck, A., Altevogt, P., Exosomes: from biogenesis and secretion to biological function. Immunol. Lett. 2006, 107, 102-108. 
[12] Pan, B. T., Johnstone, R. M., Fate of the transferrin receptor during maturation of sheep reticulocytes in vitro: selective externalization of the receptor. Cell 1983, 33, 967-978.

[13] Fevrier, B., Raposo, G., Exosomes: endosomal-derived vesicles shipping extracellular messages. Curr. Opin. Cell Biol. 2004, 16, 415-421.

[14] Ludwig, A. K., Giebel, B., Exosomes: small vesicles participating in intercellular communication. Int. J. Biochem. Cell Biol. 2012, 44, 11-15.

[15] Feng, D., Zhao, W. L., Ye, Y. Y., Bai, X. C. et al., Cellular internalization of exosomes occurs through phagocytosis. Traffic $2010,11,675-687$.

[16] Mathivanan, S., Lim, J. W., Tauro, B. J., Ji, H. et al., Proteomics analysis of A33 immunoaffinity-purified exosomes released from the human colon tumor cell line LIM1215 reveals a tissue-specific protein signature. Mol. Cell. Proteomics 2010, 9, 197-208.

[17] Simons, M., Raposo, G., Exosomes-vesicular carriers for intercellular communication. Curr. Opin. Cell Biol. 2009, 21, 575-581.

[18] Zhang, H. G., Grizzle, W. E., Exosomes and cancer: a newly described pathway of immune suppression. Clin. Cancer Res. 2011, 17, 959-964.

[19] Al-Nedawi, K., Meehan, B., Rak, J., Microvesicles: messengers and mediators of tumor progression. Cell Cycle 2009, 8, 2014-2018.

[20] Ratajczak, J., Wysoczynski, M., Hayek, F., JanowskaWieczorek, A. et al., Membrane-derived microvesicles: important and underappreciated mediators of cell-to-cell communication. Leukemia 2006, 20, 1487-1495.

[21] Valenti, R., Huber, V., Filipazzi, P., Pilla, L. et al., Human tumor-released microvesicles promote the differentiation of myeloid cells with transforming growth factor-betamediated suppressive activity on T lymphocytes. Cancer Res. 2006, 66, 9290-9298.

[22] Zomer, A., Vendrig, T., Hopmans, E. S., van Eijndhoven, M. et al., Exosomes: fit to deliver small RNA. Commun. Integr. Biol. 2010, 3, 447-450.

[23] Andre, F., Schartz, N. E., Chaput, N., Flament, C. et al., Tumorderived exosomes: a new source of tumor rejection antigens. Vaccine 2002, 20(Suppl 4), A28-A31.

[24] Dolo, V., Adobati, E., Canevari, S., Picone, M. A. et al., Membrane vesicles shed into the extracellular medium by human breast carcinoma cells carry tumor-associated surface antigens. Clin. Exp. Metastasis 1995, 13, 277-286.

[25] Hegmans, J. P., Bard, M. P., Hemmes, A., Luider, T. M. et al., Proteomic analysis of exosomes secreted by human mesothelioma cells. Am. J. Pathol. 2004, 164, 1807-1815.

[26] Mears, R., Craven, R. A., Hanrahan, S., Totty, N. et al., Proteomic analysis of melanoma-derived exosomes by twodimensional polyacrylamide gel electrophoresis and mass spectrometry. Proteomics 2004, 4, 4019-4031.

[27] Cho, J. A., Yeo, D. J., Son, H. Y., Kim, H. W. et al., Exosomes: a new delivery system for tumor antigens in cancer immunotherapy. Int. J. Cancer 2005, 114, 613-622.
[28] Filipazzi, P., Burdek, M., Villa, A., Rivoltini, L. et al., Recent advances on the role of tumor exosomes in immunosuppression and disease progression. Semin. Cancer Biol. 2012, 22, 342-349.

[29] Yang, C., Robbins, P. D., The roles of tumor-derived exosomes in cancer pathogenesis. Clin. Dev. Immunol. 2011, doi:10.1155/2011/842849.

[30] Peinado, H., Lavotshkin, S., Lyden, D., The secreted factors responsible for pre-metastatic niche formation: old sayings and new thoughts. Semin. Cancer Biol. 2011, 21, 139-146.

[31] Safaei, R., Larson, B. J., Cheng, T. C., Gibson, M. A. et al., Abnormal lysosomal trafficking and enhanced exosomal export of cisplatin in drug-resistant human ovarian carcinoma cells. Mol. Cancer Ther. 2005, 4, 1595-1604.

[32] Battke, C., Ruiss, R., Welsch, U., Wimberger, P. et al., Tumour exosomes inhibit binding of tumour-reactive antibodies to tumour cells and reduce ADCC. Cancer Immunol. Immunother. 2011, 60, 639-648.

[33] Kogure, T., Lin, W. L., Yan, I. K., Braconi, C. et al., Intercellular nanovesicle-mediated microRNA transfer: a mechanism of environmental modulation of hepatocellular cancer cell growth. Hepatology 2011, 54, 1237-1248.

[34] Al-Nedawi, K., Meehan, B., Micallef, J., Lhotak, V. et al., Intercellular transfer of the oncogenic receptor EGFRvIll by microvesicles derived from tumour cells. Nat. Cell Biol. 2008, $10,619-624$.

[35] Khan, S., Jutzy, J. M., Aspe, J. R., McGregor, D. W. et al., Survivin is released from cancer cells via exosomes. Apoptosis 2011, 16, 1-12.

[36] Webber, J., Steadman, R., Mason, M. D., Tabi, Z. et al., Cancer exosomes trigger fibroblast to myofibroblast differentiation. Cancer Res. 2010, 70, 9621-9630.

[37] Sidhu, S. S., Mengistab, A. T., Tauscher, A. N., LaVail, J. et al., The microvesicle as a vehicle for EMMPRIN in tumor-stromal interactions. Oncogene 2004, 23, 956-963.

[38] Dolo, V., Ginestra, A., Cassara, D., Violini, S. et al., Selective localization of matrix metalloproteinase 9, beta1 integrins, and human lymphocyte antigen class I molecules on membrane vesicles shed by $8701-\mathrm{BC}$ breast carcinoma cells. Cancer Res. 1998, 58, 4468-4474.

[39] Ginestra, A., Miceli, D., Dolo, V., Romano, F. M. et al., Membrane vesicles in ovarian cancer fluids: a new potential marker. Anticancer Res. 1999, 19, 3439-3445.

[40] Giusti, I., D’Ascenzo, S., Millimaggi, D., Taraboletti, G. et al., Cathepsin $\mathrm{B}$ mediates the $\mathrm{pH}$-dependent proinvasive activity of tumor-shed microvesicles. Neoplasia 2008, 10, 481-488.

[41] Escola, J. M., Kleijmeer, M. J., Stoorvogel, W., Griffith, J. M. et al., Selective enrichment of tetraspan proteins on the internal vesicles of multivesicular endosomes and on exosomes secreted by human B-lymphocytes. J. Biol. Chem. 1998, 273, 20121-20127.

[42] Charrin, S., le Naour, F., Silvie, O., Milhiet, P. E. et al., Lateral organization of membrane proteins: tetraspanins spin their web. Biochem. J. 2009, 420, 133-154.

[43] Berditchevski, F., Complexes of tetraspanins with integrins: more than meets the eye. J. Cell Sci. 2001, 114, 4143-4151. 
[44] Hemler, M. E., Tetraspanin proteins mediate cellular penetration, invasion, and fusion events and define a novel type of membrane microdomain. Annu. Rev. Cell Dev. Biol. 2003, $19,397-422$.

[45] Gesierich, S., Berezovskiy, I., Ryschich, E., Zoller, M., Systemic induction of the angiogenesis switch by the tetraspanin D6.1A/CO-029. Cancer Res. 2006, 66, 7083-7094.

[46] Pant, S., Hilton, H., Burczynski, M. E., The multifaceted exosome: biogenesis, role in normal and aberrant cellular function, and frontiers for pharmacological and biomarker opportunities. Biochem. Pharmacol. 2012, 83, 1484-1494.

[47] Hood, J. L., Pan, H., Lanza, G. M., Wickline, S. A., Paracrine induction of endothelium by tumor exosomes. Lab Invest. 2009, 89, 1317-1328.

[48] Taverna, S., Flugy, A., Saieva, L., Kohn, E. C. et al., Role of exosomes released by chronic myelogenous leukemia cells in angiogenesis. Int. J. Cancer 2012, 130, 2033-2043.

[49] Rivoltini, L., Canese, P., Huber, V., lero, M. et al., Escape strategies and reasons for failure in the interaction between tumour cells and the immune system: how can we tilt the balance towards immune-mediated cancer control? Expert Opin. Biol. Ther. 2005, 5, 463-476.

[50] Valenti, R., Huber, V., lero, M., Filipazzi, P. et al., Tumorreleased microvesicles as vehicles of immunosuppression. Cancer Res. 2007, 67, 2912-2915.

[51] Andreola, G., Rivoltini, L., Castelli, C., Huber, V. et al., Induction of lymphocyte apoptosis by tumor cell secretion of FasL-bearing microvesicles. J. Exp. Med. 2002, 195, 1303-1316.

[52] Liu, C., Yu, S., Zinn, K., Wang, J. et al., Murine mammary carcinoma exosomes promote tumor growth by suppression of NK cell function. J. Immunol. 2006, 176, 1375-1385.

[53] Ashiru, O., Boutet, P., Fernandez-Messina, L., AgueraGonzalez, S. et al., Natural killer cell cytotoxicity is suppressed by exposure to the human NKG2D ligand MICA*008 that is shed by tumor cells in exosomes. Cancer Res. 2010, $70,481-489$.

[54] Clayton, A., Mitchell, J. P., Court, J., Linnane, S. et al., Human tumor-derived exosomes down-modulate NKG2D expression. J. Immunol. 2008, 180, 7249-7258.

[55] Xiang, X., Poliakov, A., Liu, C., Liu, Y. et al., Induction of myeloid-derived suppressor cells by tumor exosomes. Int. J. Cancer 2009, 124, 2621-2633.

[56] Chen, K. G., Valencia, J. C., Lai, B., Zhang, G. et al., Melanosomal sequestration of cytotoxic drugs contributes to the intractability of malignant melanomas. Proc. Natl. Acad. Sci. USA 2006, 103, 9903-9907.

[57] Yan, X., Yin, J., Yao, H., Mao, N. et al., Increased expression of annexin $\mathrm{A} 3$ is a mechanism of platinum resistance in ovarian cancer. Cancer Res. 2010, 70, 1616-1624.

[58] Yin, J., Yan, X., Yao, X., Zhang, Y. et al., Secretion of annexin A3 from ovarian cancer cells and its association with platinum resistance in ovarian cancer patients. J. Cell. Mol. Med. 2012, 16, 337-348.

[59] Ramachandran, S., Palanisamy, V., Horizontal transfer of RNAs: exosomes as mediators of intercellular communication. Wiley Interdiscip. Rev. RNA 2012, 3, 286-293.

[60] Fabbri, M., Paone, A., Calore, F., Galli, R. et al., MicroRNAs bind to Toll-like receptors to induce prometastatic inflammatory response. Proc. Natl. Acad. Sci. USA 2012, 109, E2110-E2116.

[61] Umezu, T., Ohyashiki, K., Kuroda, M., Ohyashiki, J. H., Leukemia cell to endothelial cell communication via exosomal miRNAs. Oncogene 2012, doi: 10.1038/onc.2012.295.

[62] Bard, M. P., Hegmans, J. P., Hemmes, A., Luider, T. M. et al., Proteomic analysis of exosomes isolated from human malignant pleural effusions. Am. J. Respir. Cell. Mol. Biol. 2004, 31, 114-121.

[63] Choi, D. S., Park, J. O., Jang, S. C., Yoon, Y. J. et al., Proteomic analysis of microvesicles derived from human colorectal cancer ascites. Proteomics 2011, 11, 2745-2751.

[64] Simpson, R. J., Lim, J. W., Moritz, R. L., Mathivanan, S., Exosomes: proteomic insights and diagnostic potential. Expert Rev. Proteomics 2009, 6, 267-283.

[65] Welton, J. L., Khanna, S., Giles, P. J., Brennan, P. et al., Proteomics analysis of bladder cancer exosomes. Mol. Cell. Proteomics 2010, 9, 1324-1338.

[66] Xiao, Z., Blonder, J., Zhou, M., Veenstra, T. D., Proteomic analysis of extracellular matrix and vesicles. J. Proteomics 2009, 72, 34-45.

[67] Raimondo, F., Morosi, L., Chinello, C., Magni, F. et al., Advances in membranous vesicle and exosome proteomics improving biological understanding and biomarker discovery. Proteomics 2011, 11, 709-720.

[68] Li, Y., Zhang, Y., Qiu, F., Qiu, Z., Proteomic identification of exosomal LRG1: a potential urinary biomarker for detecting NSCLC. Electrophoresis 2011, 32, 1976-1983.

[69] Fontana, S., De Leo, G., Sedic, M., Alessandro, R., Proteomics in antitumor research. Drug Discov. Today Technol. 2006, 3, 441-449.

[70] Palazzolo, G., Albanese, N. N., Di Cara, G., Gygax, D. et al., Proteomic analysis of exosome-like vesicles derived from breast cancer cells. Anticancer Res. 2012, 32, 847-860.

[71] Lee, H. S., Jeong, J., Lee, K. J., Characterization of vesicles secreted from insulinoma NIT-1 cells. J. Proteome Res. 2009, 8, 2851-2862.

[72] Mann, M., Hendrickson, R. C., Pandey, A., Analysis of proteins and proteomes by mass spectrometry. Annu. Rev. Biochem. 2001, 70, 437-473.

[73] Gonzalez-Begne, M., Lu, B., Han, X., Hagen, F. K. et al., Proteomic analysis of human parotid gland exosomes by multidimensional protein identification technology (MudPIT). J. Proteome Res. 2009, 8, 1304-1314.

[74] Tauro, B. J., Greening, D. W., Mathias, R. A., Ji, H. et al., Comparison of ultracentrifugation, density gradient separation, and immunoaffinity capture methods for isolating human colon cancer cell line LIM1863-derived exosomes. Methods 2012, 56, 293-304.

[75] Choi, D. S., Lee, J. M., Park, G. W., Lim, H. W. et al., Proteomic analysis of microvesicles derived from human colorectal cancer cells. J. Proteome Res. 2007, 6, 4646-4655. 
[76] Hanahan, D., Weinberg, R. A., The hallmarks of cancer. Cell 2000, 100, 57-70.

[77] Kim, S., Takahashi, H., Lin, W. W., Descargues, P. et al., Carcinoma-produced factors activate myeloid cells through TLR2 to stimulate metastasis. Nature 2009, 457, 102-106.

[78] Hashino, J., Fukuda, Y., Oikawa, S., Nakazato, H. et al., Metastatic potential of human colorectal carcinoma SW1222 cells transfected with cDNA encoding carcinoembryonic antigen. Clin. Exp. Metastasis 1994, 12, 324-328.

[79] Thompson, J. A., Grunert, F., Zimmermann, W., Carcinoembryonic antigen gene family: molecular biology and clinical perspectives. J. Clin. Lab. Anal. 1991, 5, 344-366.

[80] Epple, L. M., Griffiths, S. G., Dechkovskaia, A. M., Dusto, N. L. et al., Medulloblastoma exosome proteomics yield functional roles for extracellular vesicles. PLoS One 2012, 7, e42064.

[81] Cox, J., Mann, M., Quantitative, high-resolution proteomics for data-driven systems biology. Annu. Rev. Biochem. 2011, 80, 273-299.
[82] Park, J. E., Tan, H. S., Datta, A., Lai, R. C. et al., Hypoxic tumor cell modulates its microenvironment to enhance angiogenic and metastatic potential by secretion of proteins and exosomes. Mol. Cell. Proteomics 2010, 9, 1085-1099.

[83] Bantscheff, M., Lemeer, S., Savitski, M. M., Kuster, B., Quantitative mass spectrometry in proteomics: critical review update from 2007 to the present. Anal. Bioanal. Chem. 2012, 404, 939-965.

[84] Cappadona, S., Baker, P. R., Cutillas, P. R., Heck, A. J. et al., Current challenges in software solutions for mass spectrometry-based quantitative proteomics. Amino Acids 2012, 43, 1087-1108.

[85] Gonzalez-Galarza, F. F., Lawless, C., Hubbard, S. J., Fan, J. et al., A critical appraisal of techniques, software packages, and standards for quantitative proteomic analysis. OMICS 2012, 16, 431-442.

[86] Choi, D. S., Yang, J. S., Choi, E. J., Jang, S. C. et al., The protein interaction network of extracellular vesicles derived from human colorectal cancer cells. J. Proteome Res. 2012, $11,1144-1151$. 